Pacific Journal of Mathematics

CHARACTERIZING FINSLER SPACES WHICH ARE
PSEUDO-RIEMANNIAN OF CONSTANT CURVATURE 


\title{
CHARACTERIZING FINSLER SPACES WHICH ARE PSEUDO-RIEMANNIAN OF CONSTANT CURVATURE
}

\author{
JOHN K. BEEM
}

Let $M$ be an indefinite Finsler space. The bisector of two points of $M$ is the set of points equidistant from these two points. A bisector is called flat if with any pair of points it contains the extremals joining this pair. In this paper it is shown that $M$ is pseudo-Riemannian of constant curvature if and only if $M$ locally has flat bisectors. Another result is that $M$ is pseudo-Riemannian of constant curvature if and only if $M$ can be reflected locally in each nonnull extremal.

1. Introduction. Blaschke [6] has shown that if $M$ is a two dimensional definite Finsler space in which the bisector of two points is an extremal then $M$ is a Riemannian space of constant curvature. Busemann [7] has shown that among his $G$-spaces the requirement that bisectors contain with each pair of points a segment joining this pair characterizes the Euclidean, hyperbolic and spherical spaces of dimension greater than one. Phadke [8] has investigated the flat bisector condition in two dimensional $G$-spaces which have a distance which is not necessarily symmetric. In [4] we have shown that a pseudoRiemannian manifold locally has flat bisectors if and only if it is a space of constant sectional curvature.

In the present paper an ordinary or definite Finsler space with a symmetric distance is considered to be a special case of an indefinite Finsler space. Consequently, our arguments are valid for definite metrics as well as nondefinite metrics. The arguments are different from those of Busemann [7] because he does not make any differentiability assumptions and since a number of his arguments do not extend to indefinite metrics.

2. Indefinite Finsler spaces. Let $M$ be an $n$ dimensional connected and paracompact differentiable manifold of class $C^{x}$. The local coordinates of a point $x$ will be denoted $x^{1}, \cdots, x^{n}$. In the tangent space $T(x)$ to $M$ at $x$ take the natural basis and let $y^{1}, \cdots, y^{n}$ denote the components of a vector $Y \in T(x)$. The coordinates of $Y$ are $(x, y)$. Let $L(x, y)$ be a continuous function defined on the tangent bundle $T(M)$ of $M$ which has the following properties:

(A) The function $L(x, y)$ is $C^{\infty}$ for all $(x, y)$ with $y \neq 0$.

(B) $L(x, k y)=k^{2} L(x, y)$ for all $k>0$. 
(C) The metric tensor $g_{i j}(x, y)=\frac{1}{2} \partial^{2} L / \partial y^{\prime} \partial y^{\prime}$ has $s$ negative eigenvalues and $n-s$ positive eigenvalues for all $(x, y)$ with $y \neq 0$.

(D) $L(x,-y)=L(x, y)$.

The function $L(x, y)$ is called the basic metric function. It corresponds to the square of the fundamental function $F(x, y)$ usually studied in definite Finsler spaces (compare [10]).

The manifold $M$ together with the basic metric function $L(x, y)$ is called an indefinite Finsler space of signature $n-2 s$. If $L(x, y)$ is replaced with $-L(x, y)$, then $M$ becomes a space of signature $2 s-n$. In the special case $s=0$ the manifold $M$ is a definite Finsler space. In this paper we do not exclude the case $s=0$.

When $M$ has a metric tensor $g_{i j}(x, y)$ which does not depend on $y$, then $M$ is called pseudo-Riemannian. A pseudo-Riemannian space is Riemannian when $s=0$ or $n$. If $M$ is $R^{n}$ and the metric tensor is constant, then $M$ is called pseudo-Euclidean.

Let $W, Y, Z$ be three tangent vectors at $x \in M$. Using the natural basis let $(x, w),(x, y)$ and $(x, z)$ be the respective coordinate representations of these vectors. The scalar product of $Y$ and $Z$ with respect to $W$ is defined by

$$
W(Y, Z)=g_{i j}(x, w) y^{i} z^{j}
$$

If $Y$ is a nonzero vector, then we say $Y$ is perpendicular to $Z$ when $Y(Y, Z)=0$. When $Y$ is perpendicular to $Z$ we write $Y \nmid Z$. This relation is not, in general, symmetric. When $M$ has dimension at least three we have shown [5] that perpendicularity is symmetric on $M$ if and only if $M$ is pseudo-Riemannian.

The norm squared of a vector $Y$ is defined by $|Y|^{2}=Y(Y, Y)$. The quantity $|Y|^{2}$ may be positive, negative or zero. A vector $Y$ with $|Y|^{2}= \pm 1$ is called a unit vector. If $|Y|^{2}=0$, then $Y$ is called a null vector. A vector is nonzero as long as it is not the origin of the tangent space at which it is attached.

The indicatrix $K(x)$ consists of all of the unit vectors in $T(x)$. The light cone $C(x)$ consists of the null vectors in $T(x)$.

If $Y \in K(x)$, then $Y+Z$ if and only if $Z$ is parallel to the tangent hyperplane to $K(x)$ at $Y$, compare $[\mathbf{1 0}$, p. 26].

3. The bisector condition. The Christoffel symbols $\gamma_{t k}^{\prime}(x, y)$ are defined in the usual way. The extremals are the solutions of the differential equations

$$
\ddot{x}^{\jmath}+\gamma_{i k}^{\prime}(x, \dot{x}) \dot{x}^{\prime} \dot{x}^{k}=0 .
$$

An extremal $x(t)$ with velocity vector of length zero is called a null extremal. 
A result of Whitehead [9] implies that for each point $x$ there is a simple convex neighborhood $U(x)$. Given two points $p$ and $q$ in $U(x)$ there is a unique extremal arc $\alpha(p, q)$ from $p$ to $q$ which lies in $U(x)$. In $U(x)$ the separation between two points $p$ and $q$ is defined by

$$
d(p, q)=\int L^{1 / 2}(x, \dot{x}) d t
$$

The integral is taken along $\alpha(p, q)$. The quantity $L^{1 / 2}(x, y)$ is either real and nonnegative or pure imaginary. Hence, $d(p, q)$ is either nonnegative or imaginary. The function $d$ is continuous on the domain $U(x) \times U(x)$. In indefinite metric spaces the local distance function $d(p, q)$ is usually only defined for points sufficiently close together.

The bisector of $p$ and $q$ with respect to $U(x)$ is defined by

$$
B(p, q)=\left\{p^{\prime} \in U(x) \mid d\left(p, p^{\prime}\right)=d\left(q, p^{\prime}\right)\right\}
$$

We say locally $M$ has flat bisectors if for each $x \in M$ there is a simple convex neighborhood $U(x)$ such that for all $p, q \in U(x)$ with $d(p, q) \neq 0$ the bisector $B(p, q)$ contains with any pair of points the extremals in $U(x)$ containing this pair.

4. The two dimensional case. In this section and the next we always assume $M$ satisfies the bisector condition. If $n=2$, then this is the assumption that $B(p, q)$ lies on an extremal of $M$.

Proposition 1. Let $M$ be a two dimensional indefinite Finsler space which locally has flat bisectors. Then $M$ is a pseudo-Riemannian space of constant curvature.

Proof. If $M$ has signature two or minus two, then the metric is definite and the proposition follows from the result of Blaschke [6] which was mentioned in the introduction.

Let $M$ have signature zero. The metric tensor must have one negative eigenvalue and one positive eigenvalue for all $(x, y)$ with $y \neq 0$. For each fixed $x \in M$, the light cone $C(X)$ consists of a finite number $m$ of lines passing through the origin of the tangent space $T(x)$. When $M$ is pseudo-Riemannian, the light cone consists of two lines. When $M$ is an indefinite Finsler space, the number of lines $m$ may be larger than two, see [2].

Let $m>2$ and let $U(x)$ be a simple convex neighborhood of $x$ such that $B(p, q)$ is flat whenever $p, q \in U(x)$ with $d(p, q) \neq 0$. Each $p \in U(x)$ has at least three distinct null directions and there are three null extremals through $p$ corresponding to these directions. At $x$, choose 
three null vectors $Y_{1}, Y_{2}$ and $Y_{3}$ such that any pair $Y_{l}, Y_{1}$ for $i \neq j$ is a linearly independent set. Since the null directions through a point vary continuously with the point, each null vector $Y_{t}$ attached at $x$ may be extended to a continuous and nonvanishing null vector field $Y_{1}$ defined on a neighborhood $W(x)$ with $W(x) \subset U(x)$. For each $p \in W(x)$, let $\alpha_{i}(p)$ where $i=1,2,3$ be a null extremal through $p$ with tangent vector $Y_{i}$ at $p$. Assume without loss of generality that $W(x)$ and the extemals $\alpha_{t}(p)$ have been chosen such that each extremal has its endpoints outside of $W(x)$. Choose $q=x$. For all $p$ sufficiently close to $q$ we have $\alpha_{i}(p) \cap$ $\alpha_{l}(q) \neq \phi$ when $i \neq j$, since the tangent to $\alpha_{i}(p)$ converges to $Y_{\imath}$ at $q$ as $p \rightarrow q$ and the tangent to $\alpha_{l}(q)$ is $Y$, at $q$. Choose a fixed $p$ with $\alpha_{i}(p) \cap \alpha_{l}(q) \neq \phi$ for $i \neq j$ and with $d(p, q) \neq 0$. Let $p_{1}=\alpha_{1}(p) \cap \alpha_{3}(q)$ and $p_{2}=\alpha_{2}(p) \cap \alpha_{3}(q)$. Since $d\left(p, p_{t}\right)=d\left(q, p_{t}\right)=0$. it follows that $p_{\imath} \in B(p, q)$ for $i=1,2$. The flat bisector condition implies $d(p, r)=$ $d(q, r)=0$ for all $r \in \alpha\left(p_{1}, p_{2}\right)$, since $\alpha\left(p_{1}, p_{2}\right)$ lies on the null extremal $\alpha_{3}(q)$. For each point $r \in \alpha\left(p_{1}, p_{2}\right)$, there is a null extremal $\alpha(p, r)$ which determines a null direction at $p$. Since $p \notin \alpha_{3}(q)$, distinct points of $\alpha\left(p_{1}, p_{2}\right)$ must determine distinct directions at $p$. This contradicts the fact that $p$ has only a finite number of null directions.

Assume that $m=2$. A two dimensional indefinite Finsler manifold for which $C(x)$ always consists of two lines has been shown to be a doubly timelike surface, see [2, p. 1038]. Doubly timelike surfaces have been studied by the author in [1]. In particular, the doubly timelike surfaces which locally satisfy the flat bisector condition have been completely characterized by Theorems (IV. 36) and (VI. 17) of [1]. These two Theorems together with the differentiability of $L(x, y)$ imply that $M$ is a pseudo-Riemannian manifold of constant curvature.

5. The bisector theorem. Let $M$ have dimension at least three and satisfy the bisector condition. If $p, q \in U(x)$ with $d(p, q) \neq 0$, let $r$ be the midpoint of $\alpha(p, q)$ so that $d(p, r)=d(q, r)$. The bisector $B(p, q)$ is a submanifold through $r$ of codimension one. This implies that $B(p, q)$ has an $n-1$ dimensional tangent space $T_{r}(B(p, q))$ at $r$. The space $T_{r}(B(p, q))$ is naturally identified with an $n-1$ dimensional linear subspace of the tangent space $T(r)$.

LEMMA 2. If $r$ is the midpoint of the nonnull extremal $\alpha(p, q)$, then $\alpha(p, q)$ is a perpendicular to $B(p, q)$ at $r$.

Proof. Let $W$ be the unit tangent to $\alpha(p, q)$ at $r$ and let $Y$ be a nonzero vector at $r$ in the hyperplane $T_{r}(B(p, q))$. Let $a(s)$ be the solution of the extremal equations such that $a^{\prime}(0)=Y$. For each $s$ (sufficiently small), let $x(t, s)$ represent the extremal $\alpha(p, a(s))$ for 
$0 \leqq t \leqq 1$. Let $\dot{x}$ denote the partial derivative of $x(t, s)$ with respect to t. Define

$$
f(x, \dot{x})=L^{1 / 2}(x, \dot{x})=\left[g_{i k} \dot{x}^{\prime} \dot{x}^{k}\right]^{1 / 2} .
$$

For each fixed $s$, the value of $f(x, \dot{x})$ is either real or pure imaginary. Define

$$
I_{1}(s)=\int f(x, \dot{x}) d t=d(p, a(s))
$$

where the integral is from $t=0$ to $t=1$. Differentiation of this equation with respect to $s$ yields

$$
I_{1}^{\prime}(s)=\int\left(\frac{\partial f}{\partial x^{j}} \frac{\partial x^{\prime}}{\partial s}+\frac{\partial f}{\partial \dot{x}^{\prime}} \frac{\partial \dot{x}^{\prime}}{\partial s}\right) d t
$$

Integrating by parts we obtain

$$
I_{1}^{\prime}(s)=\left.\frac{\partial f}{\partial \dot{x}^{j}} \frac{\partial x^{\prime}}{\partial s}\right|_{0} ^{1}+\int\left(\frac{\partial f}{\partial x^{\prime}}-\frac{d}{d t}\left(\frac{\partial f}{\partial \dot{x}^{\prime}}\right)\right)\left(\frac{\partial x^{\prime}}{\partial s}\right) d t
$$

This last integral must vanish because the Euler-Langrange equations hold along each extremal. Furthermore, the derivative of $x^{j}$ with respect to $s$ is zero at $t=0$. Hence,

$$
I_{1}^{\prime}(0)=\left.\frac{\partial f}{\partial \dot{x}^{\prime}} \frac{\partial x^{\prime}}{\partial s}\right|_{t=1}
$$

The next equation (compare [10, p. 15]) results from the homogeneous assumption (B) together with the definition (C) of the metric tensor.

$$
\frac{\partial g_{i k}}{\partial \dot{x}^{\prime}} \dot{x}^{i}=0
$$

This last equation and the definition of $f(x, \dot{x})$ imply

$$
\frac{\partial f}{\partial \dot{x}^{j}}=\frac{g_{i j} \dot{x}^{\prime}}{f(x, \dot{x})}
$$

Consequently,

$$
I_{1}^{\prime}(0)=\left.\frac{g_{i j} \dot{x}^{\prime}}{f(x, \dot{x})} \frac{\partial x^{\prime}}{\partial s}|=| W\right|^{-1} W(W, Y) .
$$


If $I_{2}(s)=d(q, a(s))$, then

$$
I_{2}^{\prime}(0)=-|W|^{-1} W(W, Y) .
$$

The fact that $a(s) \in B(p, q)$ implies $I_{1}^{\prime}(0)=I_{2}^{\prime}(0)$. This implies $W \dashv Y$ and establishes the Lemma.

LEMMA 3. Let $r$ be the midpoint of the nonnull extremal $\alpha\left(p_{1}, q_{1}\right)$. If $p, q \in \alpha\left(p_{1}, q_{1}\right)$ and $r$ is the midpoint of $\alpha(p, q)$, then $B(p, q)=B\left(p_{1}, q_{1}\right)$.

Proof. From Lemma 2 it follows that both $B(p, q)$ and $B\left(p_{1}, q_{1}\right)$ consist of the union of all extremals in $U(x)$ which pass through $r$ and have the property that $\alpha(p, q)$ is perpendicular to them at $r$.

Let $W$ and $Y$ be nonzero vectors attached at $x$ with coordinate representations $(x, w)$ and $(x, y)$ respectively. Then $W \dashv Y$ if and only if $g_{i j}(x, w) w^{i} y^{j}=0$. Since the metric tensor is nonsingular the vector $W$ is always perpendicular to a hyperplane containing the origin of $T(x)$. This hold even if $|W|^{2}=0$ (as long as $W \neq 0$ ). This hyperplane varies continuously with $W$ and may actually contain $W$.

Lemma 4. If $M$ is an indefinite Finsler space which locally has flat bisectors, then perpendicularity is symmetric on $M$.

Proof. The nonnull vectors are dense in the set of nonzero vectors and a vector $W$ is perpendicular to a hyperplane which varies continuously with $W$. Consequently, it is only necessary, to verify that $W+Y$ implies $Y \dashv W$ for nonnull vectors $W$ and $Y$.

Let $\alpha(p, q)$ be a nonnull extremal with midpoint $r$ and unit tangent $W$ at $r$. Let $Y$ be a nonnull vector at $r$ with $W \dashv Y$. Using the notation of Lemma 2, we let $a(s)$ be an extremal with $a(0)=r$ and $a^{\prime}(0)=Y$. The extremal $\alpha(p, q)$ has an arclength representation $b(u)$ where $-|d(p, r)| \leqq u \leqq|d(p, r)|$ and $b^{\prime}(0)=W$. Choose some fixed $s_{0}$ different from zero and let $x(t, u)$ represent the extremal $\alpha\left(a\left(s_{0}\right), b(u)\right)$ for $0 \leqq t \leqq 1$. The partial derivative of $x$ with respect to $t$ will be denoted by $\dot{x}$. Define

$$
I_{0}(u)=\int f(x, \dot{x}) d t=d\left(a\left(s_{0}\right), b(u)\right) .
$$

The arguments used in the proof of Lemma 2 yield

$$
I_{0}^{\prime}(0)=\left.\frac{\partial f}{\partial \dot{x}^{j}} \frac{\partial x^{\prime}}{\partial u}\right|_{t=1}=|Y|^{-1} Y(Y, W) .
$$


Lemma 3 implies that $I_{0}(-u)=I_{0}(u)$. It follows that $I_{0}^{\prime}(0)=0$. Hence, $|Y|^{-1} Y(Y, W)=0$. This implies $Y \dashv W$ and establishes the Lemma.

THEOREM 5. Let $M$ be an indefinite Finsler space. Locally $M$ has flat bisectors if and only if $M$ is pseudo-Riemannian of constant sectional curvature.

Proof. If $M$ has dimension two, then Proposition 1 yields the result.

In [5] we have shown that an indefinite Finsler space of dimension at least three has symmetric perpendicularity if and only if it is pseudoRiemannian. In [4] we have shown that a pseudo-Riemannian manifold locally has flat bisectors if and only if it is a space of constant curvature. These two results together with the conclusion of Lemma 4 that $M$ has symmetric perpendicularity complete the proof of the Theorem.

6. Reflections in extremals. In this section another theorem characterizing pseudo-Riemannian spaces of constant curvature is proven.

Let $f$ be a diffeomorphism of $M$ onto itself and let $f_{*}$ denote the derivative map induced on the tangent bundle. The map $f$ is an isometry if for all $x \in M$ and $W, Y, Z \in T(x)$ we have

$$
W(Y, Z)=f_{*}(W)\left(f_{*} Y, f_{*} Z\right) .
$$

When $f$ is a diffeomorphism of some open set $U_{1}$ of $M$ onto an open set $U_{2}$ of $M$ which satisfies the above equality, the map $f$ is called a local isometry. When $f$ is a local isometry different from the identity and such that $f^{2}$ is the identity, then $f$ is an involution.

Let $x$ be an interior point of the nonnull extremal $\alpha$. A reflection in $\alpha$ near $x$ is said to exist, if there is a neighborhood $V(x)$ and a local isometry $f$ defined on $V(x)$ such that $f$ is an involution and the set of fixed points of $f$ is exactly $\alpha \cap V(x)$.

If every nonnull extremal may be reflected near each interior point, then we say $M$ may be locally reflected in each nonnull extremal.

Let $f$ be a reflection in $\alpha$ near $x$. The tangent map $f_{*}$ is a linear map of $T(x)$ onto $T(x)$ which preserves the metric induced on $T(x)$. Hence, $f_{*}$ maps the indicatrix $K(x)$ onto itself and the light cone $C(x)$ onto itself. If $W$ is a nonzero vector tangent to $\alpha$ at $x$, then $f_{*} W=W$ and

$$
W(W, Z)=W\left(W, f_{*} Z\right)
$$

for all $Z \in T(x)$. This implies that if $W$ is perpendicular to the $(n-1)$ dimensional linear subspace $H$ of $T(x)$ then $f_{*} H=H$. 
Let $(M, g)$ be a pseudo-Riemannian space of constant sectional curvature. It is known (see [11, p. 69]) that each $x \in M$ must have a neighborhood which is isometric to an open set of one of the model spaces $S_{s}^{n}, R_{s}^{n}$ or $H_{s}^{n}$. When $s=0$, these model spaces are the classical models for spaces of constant curvature. The space $S_{0}^{n}$ is an $n$ dimensional sphere, the space $R_{0}^{n}$ is $n$ dimensional Euclidean space and $H_{0}^{n}$ is an $n$ dimensional hyperbolic space. The groups of motions of all of the model spaces are well known, compare [11, pp. 65-66]. In particular, each of the model spaces may be reflected over any nonnull geodesic $G$. This reflection may have more than $G$ as its set of fixed points, however, the geodesic $G$ will have a neighborhood $U$ such that the fixed points of $U$ are all on $G$. If follows that any pseudo-Riemannian space of constant curvature may be locally reflected in any nonnull extemal. In general, pseudo-Riemannian spaces of constant curvature cannot be reflected over null extremals.

Proposition 6. If $M$ is a two dimensional indefinite Finsler space which may be locally reflected in all nonnull extremals, then $M$ is pseudo-Riemannian of constant curvature.

Proof. If the metric on $M$ is definite the result is well known, see [7, p. 350].

Assume the metric is not definite and let $W$ be a nonnull vector in $T(x)$. There is a local reflection $f$ in the extremal $\alpha$ determined by $W$. Furthermore, $f_{*} W=W$ and $f_{*}$ is an involutoric motion on $T(x)$. Letting $W$ vary, it follows that there exist infinitely many motions of $T(x)$ holding the origin fixed. The metric on $T(x)$ is Minkowskian and it is known [3, p. 533] that a two dimensional Minkowskian space has an infinite group of motions holding one point fixed if and only if the metric is the ordinary two dimensional Lorentz metric. Letting $x$ vary, it follows that $M$ is pseudo-Riemannian.

Let $\alpha(p, q)$ be a nonnull extemal from $p$ to $q$. For each positive integer $k$, there is a set of equally spaced points $\left\{p_{0}, p_{\imath}, \cdots, p_{k}\right\}$ on $\alpha(p, q)$ with $d .\left(p, p_{m}\right)=m d(p, q) / k$ where $m=1,2, \cdots, k$. Each extremal $\alpha\left(p_{i}, p_{t+1}\right)$ has a midpoint $r_{i}$. Let $\alpha^{\perp}\left(r_{i}\right)$ be the nonnull extremal perpendicular at $r_{t}$ to $\alpha\left(p_{t}, p_{t+1}\right)$. Let $F_{t}$ be the local reflection over $\alpha^{\perp}\left(r_{t}\right)$. The map $F_{t}$ takes points of $\alpha\left(p_{t}, p_{t+1}\right)$ to points of $\alpha\left(p_{t}, p_{t+1}\right)$. For sufficiently large $k$ each $F_{1}$ may be defined on all of $\alpha\left(p_{i}, p_{i+1}\right)$ and this map interchanges $p_{\imath}$ and $p_{\imath+1}$. Consequently, the composite map

$$
F=F_{k} \circ F_{k-1} \circ \cdots \circ F_{1}
$$


is a local isometry taking $p$ to $q$ whenever $k$ is sufficiently large. It follows that $M$ has the same curvature at $p$ and $q$.

To conclude that $M$ has the same curvature at all points we observe that any pair of points of $M$ may be joined by a path consisting of a finite sequence of nonnull extremals. This establishes the Proposition.

LEMMA 7. Let $W$ be a unit vector at $x$ which is tangent to $\alpha$ and let $f$ be a reflection in $\alpha$ near $x$. Then $W \dashv Z$ implies $f_{*} Z=-Z$.

Proof. Let $W$ be perpendicular to $Z$. Then $W$ is also perpendicular to $f_{*} Z$ since $f_{*}$ preserves the metric on $T(x)$. Assume $f_{*} Z \neq-Z$ and let $Y=Z+f_{*} Z$. Then $Y$ is nonzero. Also, $f_{*} Y=f_{*} Z+f_{*}^{2} Z=$ $f_{*} Z+Z=Y$ and $W+Y$.

If $|Y|^{2} \neq 0$, let $\beta$ be the extremal through $x$ with tangent $Y$ at $x$. Then $f$ leaves $\beta$ pointwise fixed near $x$ which contradicts the assumption that $f$ only leaves $\alpha \cap V(x)$ fixed.

If $|Y|^{2}=0$, let $P$ be the two dimensional linear subspace of $T(x)$ spanned by $Y$ and $W$. The map $f_{*}$ is the identity on $P$ since $f_{*} Y=Y$ and $f_{*} W=W$. For sufficiently small positive $\epsilon$, the vector $X=W+\epsilon Y$ is a nonnull vector in $P$. Letting $\beta$ be an extremal tangent to $X$ at $x$, it follows as before that $f$ leaves $\beta$ pointwise fixed near $x$. This last contradiction establishes the Lemma.

THEOREM 8. If $M$ is an indefinite Finsler space, then $M$ may be reflected locally in each nonnull extremal if and only if $M$ is a pseudoRiemannian space of constant curvature.

Proof. Because of Proposition 6, we only consider $n \geqq 3$.

Let $W$ be a nonnull vector tangent to $\alpha$ at $x$. Assume that $f$ is a local reflection in $\alpha$ and that $Z$ is any vector with $W \dashv Z$. Let $(x, w)$ and $(x, z)$ be the respective coordinate representations of $W$ and $Z$. Lemma 7 and the fact that $f_{*}$ must preserve the metric induced on the tangent space $T(x)$ yield $g_{v}(x, w+\epsilon z)=g_{\imath}(x, w-\epsilon z)$ for all real $\epsilon$. This implies the derivative of $g_{i j}(x, w+\epsilon z)$ with respect to $\epsilon$ must vanish at $\epsilon=0$. The function $g_{i j}(x, y)$ is homogeneous of degree zero in $y$ because of conditions (B) and (C). Thus, the derivative of $g_{i j}(x, w+\epsilon w)$ with respect to $\epsilon$ must vanish at $\epsilon=0$. We conclude that

$$
\frac{\partial g_{i \nu}(x, w)}{\partial \dot{x}^{k}}=0
$$

for all $k=1,2, \cdots, n$. This equation must hold for all nonnull vectors $W$. 
Since the nonnull vectors at $x$ are dense in $T(x)$, we find $g_{i j}(x, \dot{x})$ is independent of $\dot{x}$. Hence, $M$ is pseudo-Riemannian.

Consider a nondegenerate two dimensional linear subspace $E$ of $T(x)$ with sectional curvature $K(x, E)$. Let $E$ be spanned by vectors $Y$ and $Z$. The two dimensional sections of $T(x)$ have a natural topology induced from the Grassmann manifold of 2-planes in $T(x)$. If $Y_{1} \rightarrow Y$ and $Z_{l} \rightarrow Z$, then the subspace spanned by $Y_{\imath}$ and $Z_{\imath}$ converges to $E$.

If $f$ is the reflection in the nonnull extremal $\alpha$ through $x$, then $K(x, E)=K\left(x, f_{*} E\right)$. In general, given two arbitrary sections $E_{1}$ and $E_{2}$ at $x$ there may not be a reflection $f$ such that $E_{2}=f_{*} E_{1}$. In fact, it may happen that the metric is definite on one section and indefinite on the other.

Let $Y^{\prime}$ be a vector attached at $x$ and let $E^{\prime}$ denote the section spanned by $Y^{\prime}$ and $Z$. If $Y^{\prime}$ is chosen sufficiently close to $Y$, then there is a reflection $f$ in some nonnull extremal $\alpha$ such that $E^{\prime}=f_{*} E$. It follows easily that all sections sufficiently close to $E$ have the same curvature. This implies that two nondegenerate sections $E_{1}$ and $E_{2}$ will have the same curvature if there is a continuous family of nondegenerate sections from $E_{1}$ to $E_{2}$. It follows that the sectional curvature $K(x, E)$ is independent of $E$. However, when $n \geqq 3$ the sectional curvature is only constant at each $x$ when the curvature is independent of $x$, see $[11, \mathrm{p} .57]$. Therefore, $M$ is a space of constant curvature.

Theorems 5 and 8 yield our final Proposition.

Proposition 9. If $M$ is an indefinite Finsler space, then the following conditions are equivalent.

(i) $\quad M$ is pseudo-Riemannian of constant curvature.

(ii) Locally $M$ has flat bisectors.

(iii) $M$ may be reflect locally in each nonnull extremal.

Remark. If $M$ has a definite Finsler metric, then Theorems 5 and 8 may be established without using the assumption of condition (D) that the metric be symmetric. Furthermore, by making some modifications of the arguments in [3] and in the proof of Theorem 8, we may establish Theorem 8 for indefinite metrics without assuming condition (D).

\section{REFERENCES}

1. J. K. Beem and P. Y. Woo, Doubly timelike surfaces, Mem. Amer. Math. Soc. No. 92, 1969.

2. - Indefinite Finsler spaces and timelike spaces, Canad. J. Math., 22 (1970), 1035-1039.

3. Motions in two dimensional indefinite Finsler spaces, Indiana Univ. Math. J., 21 (1971), $551-555$.

4. - Pseudo-Riemannian manifolds with totally geodesic bisectors, Proc. Amer. Math. Soc., 49 (1975), 212-215. 
5. - Symmetric perpendicularity for indefinite Finsler metrics on Hilbert manifolds, Geometriae Dedicata, 4 (1975), 45-49.

6. W. Blaschke, Geometrische Untersuchungen zur Variatıonsrechnung, I. Über Symmetralen, Math. Z., 6 (1920), 281-285.

7. H. Busemann, The Geometry of Geodesics, New York, Academic Press, 1955.

8. B. B. Phadke, Flatness of bisectors and the symmetry of distance, J. Geometry, 4 (1974), 35-51.

9. J. H. C. Whitehead, Convex regions in the geometry of paths - addendum, Quart. J. Math. Oxford Ser., 4 (1933), 226-227.

10. H. Rund, The Differential Geometry of Finsler Spaces, Berlin, Springer-Verlag, 1959.

11. J. A. Wolf, Spaces of Constant Curvature, McGraw-Hill, 1967.

Received July 9, 1974 and in revised form January 30, 1976.

University of Missouri-Columbia 




\section{Pacific Journal of Mathematics}

Vol. 64, No. 1

May, 1976

Walter Allegretto, Nonoscillation theory of elliptic equations of order $2 n \ldots \ldots \quad 1$

Bruce Allem Anderson, Sequencings and starters.................. 17

Friedrich-Wilhelm Bauer, A shape theory with singular homology .......... 25

John Kelly Beem, Characterizing Finsler spaces which are

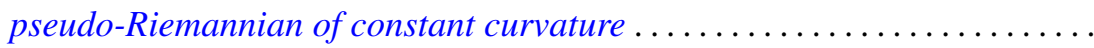

Dennis K. Burke and Ernest A. Michael, On certain point-countable

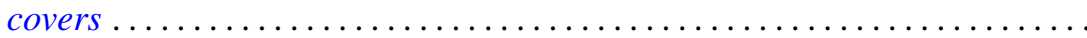

Robert Chen, A generalization of a theorem of Chacon ............... 93

Francis H. Clarke, On the inverse function theorem ................ 97

James Bryan Collier, The dual of a space with the Radon-Nikodým

property ....................................... 103

John E. Cruthirds, Infinite Galois theory for commutative rings ............ 107

Artatrana Dash, Joint essential spectra......................... 119

Robert M. DeVos, Subsequences and rearrangements of sequences in FK

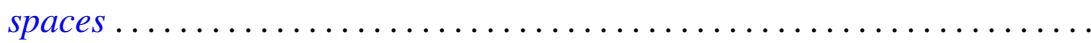

Geoffrey Fox and Pedro Morales, Non-Hausdorff multifunction generalization

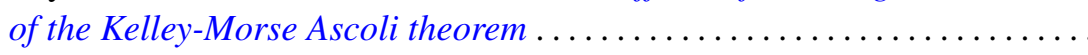

Richard Joseph Fleming, Jerome A. Goldstein and James E. Jamison, One

parameter groups of isometries on certain Banach spaces.............

Robert David Gulliver, II, Finiteness of the ramified set for branched

immersions of surfaces

Kenneth Hardy and István Juhász, Normality and the weak cb property ...... 167

C. A. Hayes, Derivation of the integrals of $L^{(q)}$-functions.

Frederic Timothy Howard, Roots of the Euler polynomials .

Robert Edward Jamison, II, Richard O'Brien and Peter Drummond Taylor, On

embedding a compact convex set into a locally convex topological vector space ....................................

Andrew Lelek, An example of a simple triod with surjective span smaller than span ...

Janet E. Mills, Certain congruences on orthodox semigroups

Donald J. Newman and A. R. Reddy, Rational approximation of $e^{-x}$ on the positive real axis.

John Robert Quine, Jr., Homotopies and intersection sequences ...

Nambury Sitarama Raju, Periodic Jacobi-Perron algorithms and fundamental units ....

Herbert Silverman, Convexity theorems for subclasses of univalent functions. . .

Charles Frederick Wells, Centralizers of transitive semigroup actions and endomorphisms of trees.........................

Volker Wrobel, Spectral approximation theorems in locally convex spaces ..................... 